\title{
LA INVISIBILIDAD DEL BIOBÍO: MIRANDO EL CENTRO DESDE LA PERIFERIA*
}

\author{
THE INVISIBILITY OF THE BIOBÍO REGION: \\ A LOOK FROM OUTSIDE
}

\section{CRISTIÁN MEDINA VALVERDE*, CRISTIÁN GUZMÁN}

\section{RESUMEN}

El estudio del desempeño económico de las regiones de Chile es un tema muy interesante que se ha hecho presente en el debate político y académico los últimos años por varias razones. Desde la óptica política una de las tesis es que el alto centralismo del país ha limitado la toma de decisiones locales, disminuyendo las posibilidades de crecimiento homogéneo. Desde la visión económica la opción de transitar hacia una economía en desarrollo ha dado pie a la discusión de las diferencias notorias en la estructura económica y social de las regiones (apoyado en la disponibilidad cada vez mayor de información económica y social de las mismas). En este contexto no deja de ser importante entonces analizar cómo se ha desempeñado censal y económicamente la Región del Biobío dentro de un contexto comparativo a nivel nacional, ya que por años fue denominada la segunda región más importante de Chile, y qué tan presente o no se encuentra frente al Estado. La visibilidad regional ha estado en el último tiempo en la base de una cierta retórica discursiva común que se ha escuchado en un buen número de provincias en Chile, la que ofrece singularidades propias según sea la región y en la que la conformación, distribución y posición geográfica que ocupan dentro del

* Este artículo forma parte del Proyecto FONDECYT No 1120405 (2012-2014), denominado "Entre el conflicto y el aislamiento: desarrollo, gobernabilidad y seguridad en las zonas fronterizas del norte y el sur de Chile a comienzos del siglo XXI". Agradecemos la colaboración de Consuelo Cerna en el desarrollo de este trabajo.

${ }^{*}$ Dr. en Historia. Docente investigador, Instituto de Historia, Facultad de Cs. Sociales y Humanidades, Universidad San Sebastián. Concepción, Chile. Correo electrónico: cristian.medina@ uss.cl

*** MBA, Universidad de Chile, Ingeniero Comercial, UCSC. Profesor de la Facultad de Ciencias Económicas y Administrativas de la Universidad Católica de la Santísima Concepción. Concepción, Chile. Correo electrónico: crguzman@ucsc.cl 
país (zonas extremas - frontera interna) juega un papel muy importante, aunque no exclusivo, al momento de la construcción de un imaginario regional. Sostenemos para el caso del presente trabajo que desde el Estado de Chile se articulan medidas económicas, políticas públicas, que no favorecen un modelo de desarrollo de forma homogénea.

Palabras clave: Chile, economía, Biobío, desarrollo.

\begin{abstract}
The study of the economic performance of the regions of Chile is a very interesting topic addressed in the political and academic debate over the past years for several reasons. From the political stand, one of the theses is that the high centralism of the country has limited local decisions-making reducing the possibilities of homogeneous growth. From an economic perspective, moving towards a developing economy has brought about the discussion of the well-known differences in the economic and social structure of the regions (supported by the availability of increasing economic and social information about them). In this context, it is only relevant to make a comparative analysis at a national level of the economic performance of the Biobío Region, which for many years was known as the second most important region of Chile, and its present relevance for the State. Over the past few years, regional visibility has been the basis of a certain common rhetorical discourse that has been heard in a number of Chilean provinces, and which is adapted according to the particular characteristics of a given region, its distribution, and geographical location (northern and southern ends, and border areas) in the country. These play an important, though not exclusive, role in the construction of a regional imaginary. This article claims that the Chilean State articulates economic measures and public policies which do not favor a homogeneous development model.
\end{abstract}

Keywords: Chile, economy, Biobío, economic development.

Recibido: 20.08.14. Aceptado: 28.11.16.

\title{
I. INTRODUCCIÓN
}

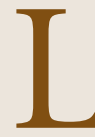

A FORMACIÓN Y CONSOLIDACIÓN de la institucionalidad del Estado chileno ha estado marcada por una trayectoria de expansión centroperiferia y el predominio de una cultura centralista como modelo de desarrollo (Boisier, 1995). El accionar estatal se ha circunscrito al Valle Central, impidiendo un desarrollo regional inclusivo. Los especialistas señalan que el " $80 \%$ de la población se asienta en 20\% del territorio" (Montero, 1998; Boisier, 2000; Montecinos, 2005). La consecuencia de ello ha sido el papel secundario asignado a las regiones donde el caso más visible es el de las zonas extremas -Aysén y Magallanes por el sur-, pero no son las únicas -Freirina y Calama por el norte-. 
En Chile el debate sobre política pública centra su análisis en el nivel macro, universal y homogéneo, ignorando la dimensión regional, sin reconocer sus especificidades históricas, geográficas, económicas, culturales, etc.

Con la globalización emergen nuevos actores y otros problemas; el Estado ha sido revalidado como eje central del desarrollo (Evans, 2003; Mittelman, 2000). La eficiencia en la generación y aplicación de las políticas públicas demanda favorecer una sociedad donde estén claros derechos y responsabilidades (Tomassini, 1998). Así el desarrollo territorial se enmarca en los procesos de modernización de la gestión pública, reforma del Estado y descentralización, emprendidos con el objetivo de alcanzar un país con una institucionalidad que responda a las demandas ciudadanas en las nuevas realidades. Es decir, la gobernabilidad es entendida como el equilibrio entre las demandas societales y la capacidad del sistema político (Estado/ gobierno) para responderlas de forma legítima y eficaz (Camou, 2001, p. 36).

La situación de las regiones de frontera interior nos interroga sobre la relación del Estado con su propio territorio, con las sociedades regionales y locales que son las que finalmente configuran la nación (Anderson, 1991).

Como bien lo señala Orrego (2007), un país desarrollado requiere de un Estado moderno que sea capaz de dar respuesta a las nuevas realidades, pero debe considerar a todos los actores que forman parte de la administración del Estado desde el nivel central hasta el nivel subnacional (regiones y municipios). Sin embargo, hay una modernización pendiente a nivel local para que los actores de este nivel sean relevantes en la agenda nacional e internacional.

El análisis de las especificidades regionales y sus dinámicas ha despertado el interés de los investigadores, particularmente en la medida en que se ha ido consolidando una sociedad más democrática; se dispone de variados canales de comunicación gracias a las nuevas redes sociales, de una mayor accesibilidad a la información y menor dependencia de los canales y fuentes oficiales (Benavente, 2009) 1 .

Por otro lado, y como se ha sostenido, "las regiones poseen dinámicas y procesos políticos particulares que son específicos a la región [y] que diferentes regiones pueden poseer patrones causales distintivos y sistemáticos que pueden ser oscurecidos por el supuesto de homogeneidad causal" (Mainwaring y Pérez-Liñán, 2009, p. 524).

${ }^{1}$ Una interpretación histórica sobre el centralismo en Chile la ofrece el historiador Guillermo Fernández (1999, p. 16). 
Los procesos de descentralización entonces plantean desafíos y problemas en el logro de los objetivos nacionales: pérdida de influencia de la capital, dispersión de cuotas reales de poder, traspaso de competencia de decisión, nuevas políticas distributivas y macroeconómicas etc. (Galdames, Krag, Menéndez, Yévenes, 2007, p. 10 ). La descentralización no sólo es un cambio político sino también cultural, lo que significa otra forma de ver la relación Estado-sociedad ${ }^{2}$. La descentralización depende de múltiples factores, como las características de cada país, su historia, los shocks externos y la capacidad para absorberlos, las dotaciones de capital y trabajo, restricciones de los gobiernos subnacionales para obtener ingresos propios. Así, por ejemplo, una descentralización fiscal óptima requiere idealmente de restricciones políticas y condiciones económicas y administrativas y mecanismos eficientes de resolución de disputas.

Esto nos incentiva a preguntarnos hasta qué punto se manifiesta la heterogeneidad de las regiones en el modelo de desarrollo en Chile. Este artículo toma como evidencia datos comparativos censales, económicos y estadísticos a nivel nacional y regional, tomando como eje central la zona del Biobío; la exposición de los datos revela la situación actual de la región y su posición socioeconómica a nivel país.

La realidad de las provincias y de las regiones se visualizó desde Santiago como una porción territorial que estaba demasiado lejos, su papel era más bien secundario y anecdótico dentro del acontecer del país. Esta visión muy extendida durante las últimas épocas aunque no del todo cierta, tuvo un giro a partir de la rearticulación del espacio político a fines del siglo XX. La literatura dedicada al desarrollo territorial y a la relación entre la institu-

${ }^{2}$ Así por ejemplo, un grupo de entidades presentó en 2013 una propuesta de "Estado Regional", verificable al 2030, y que suponía un nuevo trato Estado-territorio. La idea era luchar contra el desinterés y desactivar conflictos. Entre las medidas que se propusieron estaban: la elección popular del "Presidente regional", que reemplazaría la figura del Intendente como la cabeza del gobierno regional, en coordinación con un representante (Gobernador) del gobierno central. Instituir un ciclo de elecciones nacionales (Presidente, parlamentarios) y otras territoriales (municipales y regionales) y la residencia efectiva de los candidatos en regiones. Creación de la figura del "alcalde mayor" para administrar conurbaciones metropolitanas complejas: Santiago, Concepción-Talcahuano-San Pedro; Valparaíso-Viña del Mar. En lo económico se propuso: un Fondo de Convergencia Territorial para el desarrollo de zonas rezagadas, reformulación del Fondo Nacional de Desarrollo Regional (FNDR), además de instituir un IPC y un sueldo mínimo regional (El Sur, 21 de mayo de 2013, p. 5. Véase también, El Sur, 26 de mayo de 2013, p. 13). El tema de la autonomía regional ha sido persistente en los medios de prensa en los últimos años; a modo de ejemplo, Diario Concepción, 21 de noviembre de 2016, p. 2; Diario Concepción, 20 de noviembre de 2016, p. 2. Un hito importante fue cuando el 20 de agosto de 2015 la Presidenta Michelle Bachelet firmó el proyecto que crea la nueva Región de Nuuble, dando inicio a la discusión parlamentaria. 
cionalidad estatal y sociedades regionales y locales, concluyó que la gobernanza se presenta como una condición necesaria para lograr un desarrollo incluyente y la gobernabilidad, ya que es un enfoque que permite asociar el desarrollo económico con la institucionalidad estatal y la participación activa de la ciudadanía (OCDE, 2009, citado por De la Maza, 2010, p. 29; Mainwaring y Pérez-Liñán, 2009, p. 525).

\section{PERFIL REGIONAL DEL BIOBÍO}

El territorio representa una variable multidimensional, donde la heterogeneidad de los actores y su capacidad de agencia, las particularidades situacionales y la disponibilidad de recursos son elementos que deben ser considerados en el análisis político. Al resaltar la importancia del territorio se alude al peso que este adquiere como "constructo social", esto es, "como el resultado de un intento hecho por el individuo o un grupo de afectar, influenciar o regir a unas personas, fenómenos o relaciones, delimitando y controlando un área geográfica" (Jolly, 2003, p. 5). Desde esta perspectiva, los espacios subnacionales cobran significación e importancia como espacios de formulación e implementación de políticas públicas y del juego político.

Para el caso de Chile, el binomio gobernabilidad y territorio se ha vinculado tanto a la reforma del Estado como a la modernización de la gestión pública y a los procesos de descentralización democrática (Mardones, 2006; De la Maza, 2004 y 2010; Montecinos, 2005, 2007 y 2008, Martner, 1993, Boisier, 1995, 2000, Zárate y Artesi, 2004). Estos plantean que el desarrollo territorial es un proceso endógeno y por tanto descentralizado, en Chile esto se ha visto obstaculizado por un modelo económico de desarrollo de tipo extractivo y con un marcado centralismo político. "Esta situación no ha permitido aún el surgimiento de un nuevo modelo de desarrollo que posibilite que el territorio pueda incorporarse a las nuevas lógicas de la sociedad del conocimiento sobre la base de inclusión social, la integración territorial, la innovación y el bienestar de la comunidad" (Zárate y Artesi, 2004, p. 19).

Por lo tanto, una mirada en profundidad teórica referida a la Región del Biobío y su posición a nivel nacional permitirá constatar o no el grado de visibilidad que tiene ante el poder central.

La región cubre el 4,7\% del territorio nacional y se ubica en el extremo sur de la zona central del país. A escala nacional la Región del Biobío es 3,5 veces más pequeña que la Región de Antofagasta y 2,2 veces más grande que la Metropolitana de Santiago (RM). 
En la región habita 1.861.562 habitantes, según cifras oficiales del censo $^{3}$. En el Biobío se concentra el 12,3\% de la población del país y concentra el 7,79\% de personas perteneciente a alguna etnia. Según los datos, la zona es la segunda más poblada del país. Los datos censales indican que la tasa de crecimiento intercensal de la región tiende a disminuir, pasando de 1,6\% anual en el período 1970-1982 a 0,7\% en el período 1992-2002 (INE, 2015). Además, existe un descenso paulatino en la tasa de crecimiento de la población en la región, ya que la tasa de natalidad ha disminuido progresivamente en los últimos años, pasando de 14,2 en 2002 a 12,6 en 2013.

Si observamos la distribución de la población según regiones, más del $40 \%$ de la población del país reside en la RM, seguida por la del Biobío, $12,3 \%$, y Valparaíso, 10,40\%. Estas tres regiones concentran el mayor porcentaje de habitantes del país.

Es importante destacar que en los últimos estudios censales la Región del Biobío ocupó el tercer lugar en densidad poblacional, después de la Región Metropolitana y de Valparaíso.

En términos comparativos, la región aumentó su población en un 0,58\% (105.653 habitantes aprox.), pero con una población en franco envejecimiento (INE, 2014).

Fuera de la Región Metroplitana, Biobío tiene la mayor población con estudios de postítulo y posgrado, superando incluso a Valparaíso, que tiene mayor número de habitantes con educación superior. Entre 2002 y 2012 el porcentaje de personas con estudios superiores en la Región del Biobío creció siete puntos. Según el INE, la región pasó de 234.261 personas con educación terciaria a 388.551 , lo que significa que hoy día el $25 \%$ de los habitantes mayores de 18 cursa o cursó una carrera técnica o universitaria. La región cuenta, además, con un mayor grado de especialización que el promedio nacional, más de 16 mil personas han realizado un postítulo o posgrado, ubicándose en el primer lugar fuera del Gran Santiago ${ }^{4}$.

\footnotetext{
${ }^{3}$ En la Región del Biobío la evolución poblacional ha sido, según datos del INE: 1982: 1.517.226 habts.; 1992: 1.734.305 habts.; 2002: 1.861 .562 habts.; 2015 (proyectado): 2.114 .286 habts. Mientras que el porcentaje de variación intercensal de los períodos fue: 1982-1992: 14,0\%; 1992-2002: 7,5\%; 2002-2012: 5,7\%. Según los datos del INE (2012), ocho regiones presentan tasa de crecimiento anual mayores al promedio nacional: Tarapacá, Antofagasta, Atacama, Coquimbo, Valparaíso, O’Higgins, Metropolitana, Arica y Parinacota. Tarapacá es la región con mayor tasa de crecimiento anual en el país. Así lo revelan los censos de 1992, 2002 y 2012, siendo 2,9\%, 3,75\% y $2,37 \%$, respectivamente.

${ }^{4}$ En cuanto al número de habitantes que cursan o cursaron educación superior: Técnicos de Nivel Superior: 56.555; Profesionales: 73.701; Postítulos y Posgrados: 134.193 (Diario de Concepción, 3 de abril de 2013, p. 11). A nivel país, en 2012 se reveló que casi $1 / 3$ de los habitantes estudió
} 
Según los datos censales, Concepción es la comuna que más ha crecido en habitantes desde 2002 y se percibe una notable diferencia con el resto. El centralismo endógeno genera problemas y resentimientos, ya que la mayoría de las inversiones se centra en esta zona. El 60\% de la población regional vive sólo en 10 comunas, que son la mayoría de la Provincia de Concepción, más Chillán y Los Ángeles. Lo anterior explica la fuerte concentración y aumento de habitantes en la sección central del borde costero, en desmedro de los territorios interiores y cordilleranos, lo que ciertamente fundamenta la localización territorial en el desarrollo de infraestructura y de servicios que facilita la integración territorial de ciertas zonas de la región, en desmedro de otras (Universidad del Bío-Bío, 2009).

\section{SITUACIÓN ECONÓMICA DE LA REGIÓN DEL BIOBÍO}

La Región del Biobío es uno de los polos industriales más importantes del país, se ha consolidado como ciudad universitaria; pero su desempeño económico muestra un lento dinamismo. Su tasa de desempleo es mayor al promedio nacional y los reclamos al poder central debido a la postergación o invisibilidad de la región son latentes a nivel local.

La centralización de las decisiones de carácter económico se refleja en las cifras; sólo un porcentaje menor del total del gasto público en Chile se decide en las regiones. $\mathrm{Al}$ respecto, un destacado economista y ex ministro de Estado sostuvo que "el promedio de los países de la OCDE es de $50 \%$. La descentralización fiscal es un tema fundamental para el desarrollo y probablemente Chile sea uno de los países más centralizados del mundo. Cuando hablamos de eso, lo que realmente importa es el traspaso de poder de decisión en lo fiscal y regulatorio desde el gobierno central a gobiernos comunales y regionales"

Por su parte, 7,7\% del PIB es el porcentaje generado en la Región del Biobío en el total del PIB nacional en 2015, ocupando el cuarto lugar después de la RM, Antofagasta y Valparaíso. Hace décadas la región aportaba

en la educación superior, es decir 3,6 millones de personas, muy por encima de los 2,2 millones que había en 2002 (El Mercurio, 3 de abril de 2013, p. C 8). Mayores datos en El Sur, Posgrados, Ediciones especiales, 28 de noviembre de 2016.

${ }^{5}$ Juan Andrés Fontaine, ex ministro de Economía, académico y consultor (El Diario de Concepción, 10 de mayo de 2013, p. 12). 
alrededor de un 12 a 14\% al PIB. Esto se traduce en empobrecimiento regional ${ }^{6}$.

Así el Estado de Chile, concentrado en Santiago, no ha sido capaz de articular políticas públicas en función de las regiones, lo que se ve reflejado en la estructura productiva regional, y sostiene un modelo de desarrollo homogéneo cuyos efectos provocan una doble respuesta: profundización del regionalismo e invisibilidad de la región frente al poder central. Esto ha perpetuado en las ciudades capitales regionales la idea de su postergación por parte de la capital.

El punto de análisis es la alta concentración que se da de la actividad productiva del país en la RM. Las cifras muestran las diferencias de participación entre las tres regiones más importantes en aporte al PIB después de la RM, y la producción de esa es abismal. Mientras las regiones de Antofagasta, Valparaíso y Biobío aportan en promedio el 9\% del PIB regionalizado, la RM concentra cerca del 50\% de la producción nacional regional. Además se puede apreciar cómo el resto de las regiones no alcanza a aportar el 50\% de lo que aporta la RM. Este hecho unido a otros de decisión están provocando diferencias importantes entre las regiones del país, sobre todo que el alto centralismo de las decisiones implica la aplicación de políticas públicas transversales al país sin reconocer la especificidad regional.

La falta de comprensión de la realidad regional chilena con el centro del país ha desarrollado un imaginario que castiga a las regiones, las que se sienten en un plano de desventaja frente al poder político central. El gráfico PIB 2003-2010, que apoya nuestra afirmación, revela las asimetrías regionales y evidencia la preeminencia de la capital. En 2012 el crecimiento del PIB fue de un 5,6\%, así que Chile lideró el crecimiento entre países OCDE y fue segundo en Sudamérica.

\footnotetext{
6 "Casi todos los estudios muestran que el nivel de desarrollo económico, generalmente operacionalizado por el ingreso per cápita, es un poderoso predictor de democracia (...) La mayor parte $(. .$.$) asume que el nivel de desarrollo tiene un impacto regionalmente uniforme sobre la$ democracia" (Mainwaring y Pérez-Liñán, 2009, p. 528).
} 


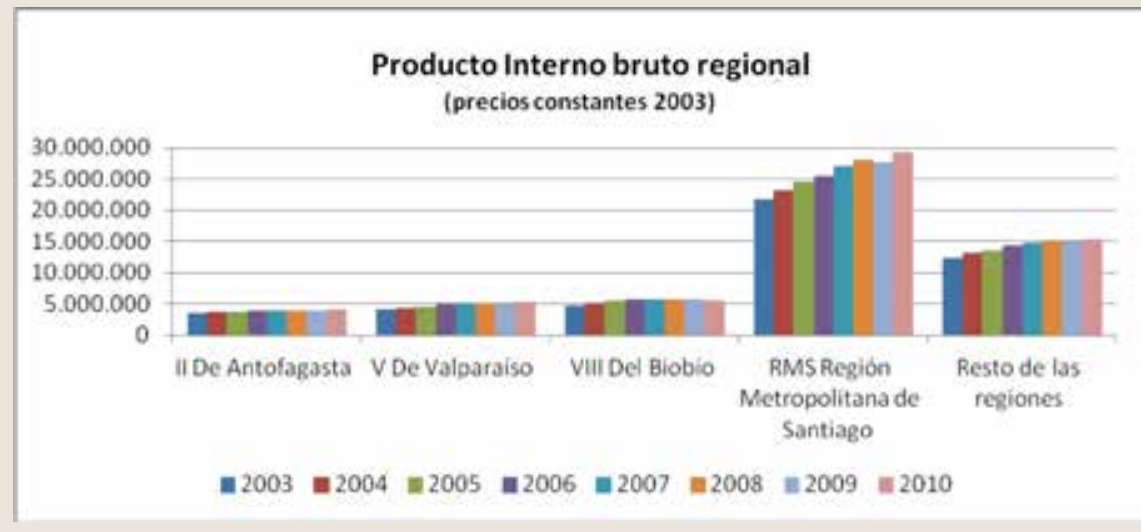

Fuente: Elaboración propia en base a datos del Banco Central.

Las auspiciosas cifras macroeconómicas que suelen darse contrastan con las que exhibe la Región del Biobío, que hoy crece a la mitad del promedio nacional. La participación de la zona ha decaído en las dos últimas décadas, y hoy es la segunda región más pobre de Chile. No obstante, las cifras de crecimiento y cesantía contrastan con las virtudes que posee la región ${ }^{7}$. Una de las causas de por qué las regiones, y en especial la del Biobío, no muestran mayor dinamismo es la alta concentración de actividad económica en la RM, que implica concentración de capital humano y físico.

La región presenta los peores rendimientos en materias de actividad económica, empleo y desarrollo social, cuyo corolario es un estado de permanente postergación económica.

Para que el sector privado aproveche las oportunidades que ofrece la expansión económica del país, se requiere de un fuerte compromiso del Estado para estimular la inversión. También se necesita innovación, desarrollo de ciencia y tecnología, que agreguen valor a la exportación de productos regionales. Al respecto, el editorial de un importante periódico de la zona señaló hace un tiempo que: "En la medida que no haya convergencia en la estrategia de desarrollo regional, deberemos acostumbrarnos a ser simples espectadores del desarrollo económico y social de Chile".

\footnotetext{
${ }^{7}$ Entre ellas: plantaciones forestales, sector pesquero, industria pesada, clima privilegiado para la producción agrícola, vitivinícola, ganadera, etc., buena infraestructura educacional en todos los niveles, es una de las regiones de mayor aporte energético al Sistema Interconectado Central, buena infraestructura portuaria, buena conectividad terrestre y aérea.

${ }^{8}$ El Diario de Concepción, 10 de abril de 2013, p. 3.
} 
Ramírez y Silva (2008) clasifican a la región como estancada en el período 1990-2010, explicada por un PIB per cápita bajo el promedio nacional y una tasa de crecimiento por debajo de la tasa de crecimiento del país.

Como se puede apreciar en el gráfico Tasa de Crecimiento Anual, salvo en 2011, la tasa de crecimiento de la región ha sido menor a la tasa de crecimiento del país y, desde el año 2014, cercanas debido a la menor actividad económica del país, con una tendencia a la baja constante9. Si se compara con las vecinas regiones del Maule y de La Araucanía, ambas presentan una tasa de crecimiento promedio para el período 2010-2015 mayor a la tasa de crecimiento de la Región del Biobío ${ }^{10}$. Este efecto de menor crecimiento promedio no es sólo producto de la crisis financiera mundial de 2009 ni del terremoto de 2010, es reflejo de la caída del dinamismo regional, en comparación con las vecinas, que viene desde el 2006.

\section{Tasa de Crecimiento Anual}

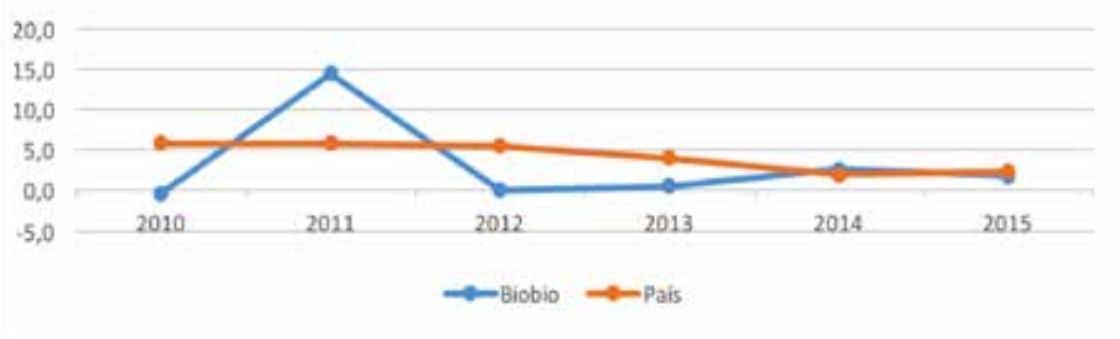

Fuente: Elaboración propia en base a los datos del Banco Central.

Otro de los efectos del lento dinamismo económico de la región es la baja participación que tiene en el PIB total del país, como muestra el siguiente gráfico para el período 2010-2015. Refleja, además, que la Región del Biobío no aporta más que el 10\% del PIB nacional, siendo la RM la que concentra cerca del $50 \%$ de la producción nacional y las regiones mineras

\footnotetext{
${ }^{9}$ Una explicación de la mayor diferencia de 2010 entre el país y la región es los efectos que produjo el terremoto en la capacidad productiva de la región.

${ }^{10}$ La Región del Maule tiene una tasa de crecimiento promedio de 3,9\%, mientras que la Región de La Araucanía tiene una tasa de 4,6\%.
} 
del norte de Chile las que más han aumentado su aporte a la producción nacional, pero sin ser más de un $10 \%$.

Producto Interno Bruto Nacional Regionalizado y Región del Biobío (millones de pesos de 2008)

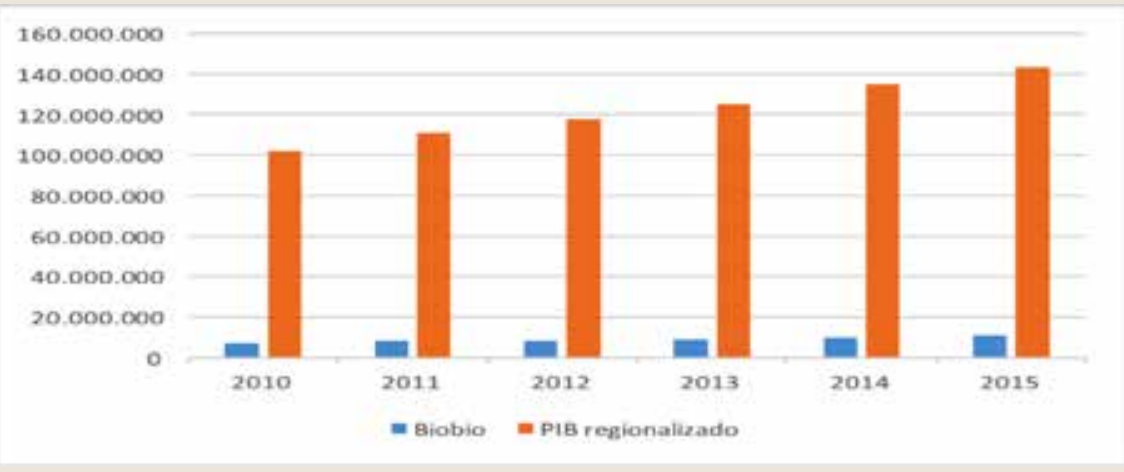

Fuente: Elaboración propia en base a datos del Banco Central.

En la tabla relativa a Actividad Económica, en la Región del Biobío existe una alta concentración de la actividad productiva en la industria manufacturera. Esta actividad económica explica más de un tercio del PIB de la región, haciendo dependiente el dinamismo de este sector. Además, este sector ha presentado una continua disminución en su tasa de crecimiento en el período 2008-2014, incluso mostrando una tasa de crecimiento negativa entre 2008 y 2009, lo que afecta el desempeño de la Región del Biobío, dada su concentración productiva ${ }^{11}$. Las causas de esta disminución en la tasa de crecimiento son varias, una de ellas es la fuerte competencia extranjera debido a la apertura comercial como estrategia de crecimiento. Sin embargo, la apertura comercial nacional ha ido en paralelo a la falta de un plan de reconversión productiva para la región. El resultado de este proceso ha llevado a generar la mayor tasa de desempleo a nivel nacional debido a la alta pérdida de empleos de este sector.

${ }^{11}$ Entre 2010 y 2015 el subsector textil, prendas de vestir, cuero y calzado ha presentado 3 años de tasa de crecimiento negativo. Sectores que fueron altamente creadores de empleo en la Región del Biobío, pero que ha disminuido su participación en la región llegando al cierre de varias plantas productivas. 
Actividad económica Región del Biobío (\%)

\begin{tabular}{|l|r|r|r|r|r|r|c|}
\hline & $\mathbf{2 0 0 8}$ & $\mathbf{2 0 0 9}$ & $\mathbf{2 0 1 0}$ & $\mathbf{2 0 1 1}$ & $\mathbf{2 0 1 2}$ & $\mathbf{2 0 1 3}$ & $\mathbf{2 0 1 4}$ \\
\hline Agropecuario-silvícola & $\mathbf{7 , 2 8}$ & $\mathbf{6 , 0 0}$ & $\mathbf{6 , 2 2}$ & $\mathbf{5 , 8 8}$ & $\mathbf{5 , 5 9}$ & 5,92 & 5,07 \\
\hline Pesca & 1,64 & 1,40 & 1,00 & 1,07 & 0,86 & 0,47 & 0,68 \\
\hline Minería & 0,09 & 0,09 & 0,07 & 0,08 & 0,06 & 0,06 & 0,08 \\
\hline Industria manufacturera & 26,23 & 25,45 & 22,09 & 23,36 & 23,74 & 23,23 & 23,15 \\
\hline Electricidad, gas y agua & 10,02 & 11,87 & 12,44 & 12,53 & 13,57 & 15,59 & 16,13 \\
\hline Construcción & 9,23 & 9,14 & 10,32 & 10,86 & 8,68 & 7,29 & 7,09 \\
\hline Comercio, restaurantes y hoteles & 7,45 & 7,10 & 8,21 & 8,10 & 8,46 & 8,64 & 8,60 \\
\hline Transportes y comunicaciones & 7,56 & 6,97 & 6,98 & 6,93 & 7,34 & 7,63 & 7,80 \\
\hline Servicios financieros y empresariales & 7,38 & 7,62 & 8,16 & 7,91 & 7,83 & 7,78 & 7,72 \\
\hline Propiedad de vivienda & 5,10 & 5,63 & 5,12 & 4,78 & 4,97 & 5,07 & 5,04 \\
\hline Servicios personales & 12,40 & 12,80 & 12,91 & 12,76 & 13,51 & 13,91 & 14,15 \\
\hline Administración pública & 5,61 & 5,93 & 6,15 & 5,42 & 5,61 & 5,80 & 5,90 \\
\hline Producto interno bruto & 100,00 & 100,00 & 100,00 & 100,00 & 100,00 & 100,00 & 100,00 \\
\hline
\end{tabular}

Fuente: Elaboración propia en base a datos del Banco Central.

El desarrollo de la región como una zona netamente industrial es una de las causas del porqué ha presentado un débil desempeño económico ${ }^{12}$. Esto sumado al cierre de actividades productivas, que, a pesar de no ser importante en aporte al PIB de la región en valor de mercado, eran generadoras de mucho empleo ${ }^{13}$.

El desempeño económico regional y sus efectos en el empleo se reflejan directamente en los indicadores de bienestar de las personas. La tasa de desempleo de la región es alta, según cifras de la CASEN, siendo 12,7\% en 2011 y $9,9 \%$ en 2013, explicada en parte por los efectos de la crisis financiera mundial y la concentración productiva de nuestra región en actividades económicas altamente dependientes de la demanda externa ${ }^{14}$. La tasa de desempleo regional es más elevada que la de la RM, además, el ingreso au-

${ }^{12}$ En Chile la industria manufacturera representa menos del 20\% del PIB.

${ }^{13}$ Las ciudades de Coronel y Talcahuano poseen las más altas tasas de desempleo de la región persistente en el tiempo. Véase por ejemplo, revista Qué Pasa, "El carbón después del carbón”, 14 de junio de 2013, pp. 40-43.

${ }^{14}$ En agosto de 2016 la tasa de desempleo de la región es de 7,7\%. Se sigue ubicando un 1\% por sobre el promedio del país. 
tónomo de la RM es 1,6 veces mayor que el de la Región del Biobío, lo que genera un desincentivo mayor a la retención del capital humano formado en la región.

El problema mayor se genera en la tasa desempleo de las mujeres; supera al promedio regional y a los hombres; además, un $40 \%$ de los hogares pobres y pobres extremos de la región tienen como jefe del hogar a una mujer. Por lo tanto, la incapacidad de generar ingresos explica la mayor pobreza presente en la región, que es mayor a la nacional y que ha sido permanente según la CASEN.

Entre 2011 y 2015 el porcentaje de hogares pobres ha presentado variaciones significativas. Mientras en la RM hubo una disminución de más de $2 \%$ en el porcentaje de hogares pobres, en la Región del Biobío en el mismo período fue cercano a un $5 \%$. Sin embargo, aún la tasa de pobreza en la Región del Biobío se encuentra sobre el promedio nacional en aproximadamente un 6\%. Este comportamiento muestra que en la Región del Biobío existen problemas estructurales diferentes al país y a la RM que necesitan la aplicación de políticas públicas con mayor focalización para la reducción de la pobreza.

\section{CONCLUSIÓN}

La hipótesis planteada se respalda en función de los datos cuantitativos entregados que muestran, entre otras cosas, que la RM concentra el $40 \%$ de la población, el $43 \%$ del PIB, en sólo un $2 \%$ del territorio nacional, situación propia de un país subdesarrollado. Si agregamos que la inversión pública es 2,8 veces más alta en la RM que en la Región del Biobío, la asimetría y la heterogeneidad regional se profundiza. Además, la región se ubica como la segunda del país con un porcentaje de población indígena relevante, situación que no supone una posición distinta frente al poder central.

Las cifras muestran que una de las debilidades de la región es su estructura productiva, que concentrada en la industria manufacturera ha tenido un dispar desempeño en los últimos 8 años.

La región cuenta con una alta tasa de matrícula en la educación terciaria pero con baja retención, ya que no existen polos de desarrollo productivo que permitan generar incentivos que capturen a los profesionales egresados de los centros de formación.

Desde la década de los noventa la región perdió competitividad. Los índices constatan la situación de condena en la que se encuentra y su falta 
de liderazgo a nivel nacional. Un tema que preocupa es el alto nivel de desempleo y la fuga de profesionales al norte y centro del país ${ }^{15}$. Baja movilidad laboral y sueldos precarios inciden en las cifras negras y son variables importantes.

El aporte de la región al PIB ha ido decayendo y esto tiene su correlato en el menor ritmo de sus avances. Vemos aquí el reflejo de la generación de políticas públicas transversales al país que se generan desde el poder central y no desde la especificidad regional.

Urge la adopción de un estado regional con autoridades elegidas, con participación en el gobierno central, con poder efectivo y asignación justa y suficiente de recursos para realizar su labor. Es decir, una mayor descentralización política y administrativa, con fortalecimiento local y regional y aumentar la participación ciudadana. Se debe avanzar en un proceso de descentralización gradual, heterogéneo y coherente que reconozca la diversidad de los territorios.

Como objetivos pendientes a nivel regional están el apoyar programas de inversión regional destinados a crear desarrollo social y económico. Pero lo importante tiene que ver con crear la necesidad de descentralizar el país, no por capricho o rivalidad, sino porque Chile no puede seguir así. La primera tarea consiste en que la Región recobre su voluntad de crecer, vuelva a su unidad de propósito para lograr, con fuerza colectiva, que los cambios se produzcan. Es un deber de Estado, es un proyecto inevitable e imprescindible como país conseguir el desarrollo armonioso de Chile.

\section{REFERENCIAS}

Anderson, B. (1991). Imagined Communities: Reflections on the origin and spreads of nationalism. London-New York: Verso.

Benavente Millán, D. J. (2009). "El centralismo latinoamericano y el desafío regionalista". Actualidad Regional, Revista de temas regionales, federalistas y desarrollo local. Disponible en: http://apunteslocales.blogspot. com/2009/03/el-centralismo-latinoamericano-y-el.html

\footnotetext{
${ }^{15}$ La tasa de desempleo en la región fue de un 7,5\%, la más alta del país, el trimestre septiembre-noviembre de 2016. Dentro de las comunas con mayor índice de cesantía Lota lidera con una tasa de $12,7 \%$ de desocupación. Coronel se ubica en el segundo lugar del ránking con una tasa más alta de 12,0\%. http://diarioconcepcion.cl/?q=content/regi\%C3\%B3n-del-b\%C3\%ADob\%C3\%ADo-lidera-cifras-de-desempleo-nivel-nacional-con-un-75
} 
Boisier, S. (1995). “El desafío territorial de la globalización. Reflexiones acerca del sistema regional chileno". Documento. Serie de Investigación ILPES No 15, Santiago, pp. 756-775.

Boisier, S. (2000). "Chile: la experiencia regionalista del gobierno militar". Estudios Sociales, 104, 43-76.

Camou, A. (2001). Los desafíos de la gobernabilidad. México D.F.: FLACSO, UNAM, Plaza y Valdés.

De la Maza, G. (2010). Mapa de actores regionales estratégicos y su articulación con el sector público para la descentralización, en tres regiones de Chile. Santiago: Innovación ciudadana. Disponible en: http://www.innovacionciudadana.cl/portal/imagen/File/descentralizacion/Sociedad/Mapa\%20 de\%20Actores\%20Regionales\%20Estrategicos.PDF

De la Maza, G.; Fernández, M.; Navarro, I. (Comps.) (2004). Innovación y ciudadanía en la gestión territorial: El rol de los municipios. Santiago: Programa de Innovación y Ciudadanía.

Evans, P. (2003). "Hybridity as an administrative Strategy: Combining bureaucratic Capacity with Market signals and Deliberative Democracy”. Revista del CLAD Reforma y Democracia, 25, 7-33.

Fernández, G. (1999). El extravío histórico chileno. Concepción: Ediciones Cerro Negro.

Galdames, R.; Krag, P.; Menéndez, J.; Yévenes, A. (2007). "Propuesta conceptual para una medición del proceso de descentralización y regionalización". Estudios Regionales, 31, 1-39. CEUR, Universidad del Bío-Bío.

INE (2014). Actualización de proyecciones de población (2002-2020). Disponible en: http://www.ine.cl/canales/sala_prensa/revistaseconomicas/presentaciones/pdf/proyecciones_poblacion_2014.pdf

INE (Instituto Nacional de Estadísticas) (2015). Evolución de la población del Biobío desde 1970 a 2020, Enfoque estadístico, noviembre.

Jolly, J. F. (2003). Lo público y lo local. Gobernancia y políticas públicas. Documentos IIGC (1-35). Barcelona: Instituto Internacional de Gobernabilidad Cataluña. Disponible en: http://www.iigov.org/biblioteca/readResource. drt?id=159, 2003.

Mainwaring, S.; Pérez-Liñán, A. (2009). “¿Por qué son importantes las regiones? Especificidades regionales y difusión de la democracia a escala regional". Revista SAAP, 3(3), agosto, 523-566.

Mardones, R. (2006). "Descentralización y transición en Chile". Revista de Ciencia Política, 26(1), 3-24.

Martner, G. (1993). Descentralización y modernización del estado en la transición. Santiago: Lom.

Mittelman, J.(2000). El síndrome de la globalización. Madrid: Siglo XXI.

Montecinos, E. (2005). "Antecedentes sobre la relación histórica centralismo y descentralización en Chile”. Revista Venezolana de Gerencia, 10(31), 443462. 
Montecinos, E. (2007). “Limitaciones del diseño institucional para una gestión municipal participativa. El caso chileno". Economía, Sociedad y Territorio, $23,725-743$.

Montecinos, E. (2008). "Los incentivos de la descentralización en la gestión municipal chilena. Gestión política sin planificación democrática". Estado, Gobierno y Gestión Pública, 12, 61-84.

Montero, P. (1998) "Chile: Nuevo concepto de fronteras interiores". Boletín de la Sociedad Geográfica de Colombia, 42(126).

Orrego, C. (2007). "Modernización del estado y gestión local". En Institucionalidad para el desarrollo: Los nuevos desafíos (pp. 55-64). Santiago: CEP y Expansiva.

Ramírez, J.; Silva, I. (2008). "Globalización y desarrollo regional, evolución económica de las regiones chilenas, 1990-2002". Revista de la Cepal, 95, agosto, 103-124.

Tomassini, L. (1998). “Gobernabilidad y políticas públicas”. En Urzúa, R. y Agüero, F. (Eds.), Fracturas en la gobernabilidad democrática (pp. 31-63). Santiago: Centro de Análisis de Políticas Públicas.

Universidad del Bío-Bío (2009). Estudio de fortalecimiento de la identidad regional. Región del Biobío, julio, Concepción, 181 pp.

Zárate, R. y Artesi, L. (2004). Conocimiento, periferia y desarrollo. Los nuevos escenarios de la Patagonia Austral. Buenos Aires: Editorial Biblos, 344 pp. 